Revue d'histoire de l'Amérique française

BHS REVUE D.HISTOIRE DE L'AMÉRIQUE FRANÇAISE

\title{
Monseigneur Antoine Racine et la question universitaire canadienne (1875-1892)
}

\section{Germain Lavallée}

Volume 12, numéro 1, juin 1958

URI : https://id.erudit.org/iderudit/301885ar

DOI : https://doi.org/10.7202/301885ar

Aller au sommaire du numéro

Éditeur(s)

Institut d'histoire de l'Amérique française

ISSN

0035-2357 (imprimé)

1492-1383 (numérique)

Découvrir la revue

Citer cet article

Lavallée, G. (1958). Monseigneur Antoine Racine et la question universitaire canadienne (1875-1892). Revue d'histoire de l'Amérique française, 12(1), 80-107. https://doi.org/10.7202/301885ar d'utilisation que vous pouvez consulter en ligne.

https://apropos.erudit.org/fr/usagers/politique-dutilisation/ 


\title{
MONSEIGNEUR ANTOINE RACINE
}

\author{
ET \\ LA QUESTION UNIVERSITAIRE CANADIENNE \\ (1875-1892) \\ INTRODUCTION
}

Écrire une étude d'une certaine étendue sur le rôle qu'a joué un humble Évêque de province dans la grande question universitaire canadienne de l'autre siècle, voilà qui peut bien paraître une gageure. C'est un peu la réaction que nous avons rencontrée tant à Montréal qu'à Québec, quand nous avons exprimé le désir de présenter ce sujet. Par exemple, l'archiviste de l'Archevêché de Québec nous écrivait le 12 septembre 1953: "Il ne semble pas que Mgr Racine ait joué un grand rôle dans cette question.»

Par ailleurs, deux érudits de Sherbrooke nous encourageaient à explorer ce sujet: l'abbé Maurice O'Bready, qui travaille à l'histoire des Cantons de l'Est et l'abbé Albert Gravel, auteur d'une histoire religieuse du diocèse de Sherbrooke et d'articles dans Le Messager Saint-Michel, journal du même diocèse.

Fort de ces encouragements, nous sommes partis à la chasse des documents et - faut-il le dire - nous avons rapporté un abondant gibier. Les Archives de l'Archevêché de Sherbrooke nous ont fourni environ le tiers de notre documentation, les deux tiers étant extraits des Archives du Séminaire de Québec et de l'Université Laval, ainsi que de la collection publiée par le Chanoine Jean-Baptiste Proulx, Vice-Recteur de l'Université Laval à Montréal. Nous tenons à ajouter combien les conseils de Mgr Émile Chartier, professeur émérite de l'Université de Montréal, nous ont été précieux.

Nous n'avons pas la prétention d'avoir épuisé notre sujet. Nous croyons toutefois avoir suffisamment élaboré et dépouillé 
notre documentation pour offrir un texte historique qui dépasse souvent l'hypothèse.

Notre travail tâchera d'expliquer les deux attitudes paradoxales de Mgr A. Racine dans la question universitaire:

a) son opposition déclarée à la fondation d'une université catholique à Montréal, indépendante de l'Université Laval de Québec;

b) son ardeur à stimuler le développement de la Succursale de l'Université Laval à Montréal jusqu'à annuler l'influence des Messieurs de Québec dans l'administration universitaire montréalaise.

$\mathrm{Au}$ cours de ce travail on verra que, en raison de sa belle personnalité, Mgr Racine était appelé à jouer un rôle important dans la question universitaire. La solidité de son jugement, la souplesse de son intelligence et le calme de son tempérament en faisaient un homme tout désigné pour servir d'intermédiaire entre Québec et Rome d'une part, entre Montréal et Rome ensuite. Mgr Racine fut l'avocat de deux grandes causes. Voyons comment il s'en est tiré.

Encore une fois le rôle du premier évêque de Sherbrooke pourra paraître peu considérable en ce chapitre de l'histoire canadienne. Nous croyons que toute documentation, si modeste soit-elle, mérite d'être versée au dossier de la mémorable controverse.

G.L.

Chapitre I

LE NOUVEL EVEQUE DE SHERBROOKE

Sera-t-il fidèle à son Alma Mater ? - Son passé le laisse croire Le Séminaire de Québec, un modèle - Les accusations de M. Benjamin Pâquet - Une lettre pastorale de Mgr Racine - Mgr Racine à Rome en 1877 - L'adresse du Cercle Catholique - Les soupçons durent toujours - Les résultats du voyage - Mgr Racine se réserve de la disponibilité.

Le 28 août 1874, l'illustre pontife Pie IX érigeait le diocèse de Sherbrooke, comprenant un démembrement des diocèses de Québec, de St-Hyacinthe et des Trois-Rivières, et incluant la plus 
grande partie des Cantons de l'Est. Par une bulle datée de Rome, le premier septembre de la même année, le Pape nommait le curé de St-Jean de Québec, Messire Antoine Racine, premier évêque du nouveau diocèse. Le 18 octobre suivant, le nouvel élu fut sacré par sa Grâce Monseigneur A. Taschereau, Archevêque de Québec. ${ }^{1}$

Aussitôt connue la nomination du nouvel évêque, la question ne mit pas de temps à se poser à Québec: Mgr Racine se rangerat-il parmi les partisans de l'Université Laval ou penchera-t-il plutôt vers Montréal? Le Supérieur du Séminaire de Québec, qui était en même temps le Recteur de l'Université Laval, crut d'abord que «Mgr Racine est bien disposé à notre égard ».2 Celui qui avait prononcé la principale allocution au $200^{\mathrm{e}}$ anniversaire de la fondation du Séminaire de Québec, pouvait-il se transformer aussi vite en un adversaire? Mgr Racine avait prononcé ces paroles:

Faites, ô mon Dieu, que le Séminaire de Québec soit toujours ce qu'il est aujourd'hui, un centre de lumière, le rempart de notre foi, le gardien toujours fidèle des traditions catholiques; qu'il vive et qu'il s'agrandisse autant que le veut notre amour, autant que le veut notre reconnaissance, et que ceux qui viendront après nous ... le retrouvent plus fort et plus glorieux, toujours dévoué à notre gloire, toujours dévoué à la défense de notre église, toujours dévoué à la Patrie. ${ }^{3}$

Part faite à l'éloquence et à l'enflure du style, il reste que le témoignage est sincère.

L'Évêque de Sherbrooke allait sûrement garder son amitié et son admiration au Séminaire de Québec. Quand il voudra fonder lui-même un séminaire diocésain, il s'appliquera à imiter Mgr de Laval «marchant sur les traces de l'immortel fondateur 1894).

${ }^{1}$ P.-J.-A. Lefebvre, ptre, Monseigneur Antoine Racine (Sherbrooke,

2 T.-E. Hamel, ptre, à Benjamin Pâquet, ptre, 9 octobre 1874, aux archives du Séminaire de Québec et de l'Université Laval, 110 - no BI.

Nous citerons désormais cette source: Un. 110 - no BI.

3 Mgr A. Racine à T.-E. Hamel, ptre, 7 septembre 1876, Un. $114-$ no CQ. Mgr Racine rappelle à T.-E. Hamel, un passage du discours qu'il a prononcé lors du $200^{\circ}$ Anniversaire du Séminaire de Québec. 
du Séminaire de Québec » ${ }^{4}$ pour réussir à fonder un Séminaire, qui, comme celui de Québec, sera l'asile de la vertu et de la science, la gloire du pays et l'honneur de la Religion ! ${ }^{5}$

Mais qui donc mettait en doute la bonne foi du nouvel évêque ?

Nul autre que l'abbé Benjamin Pâquet: «J'ai mis Monseigneur l'Archevêque en garde contre Mgr Racine qui est plus fourbe et plus traître que jamais. Plus tard je pourrai vous dire bien des choses à ce sujet ! ${ }^{6}$ Puis il ajoute: «En attendant sachez, mais, gardez-le pour vous, que Mgr Racine partage absolument toutes les opinions de M. A. Pelletier! $\gg^{7}$ Pourtant Mgr Racine est un homme pacifique: «Espérons, avec M. le Recteur, écrit-il, que la Bulle «Inter varias sollicitudines » sera un signal de ralliement qui fera cesser les malentendus. Malentendus regrettables à l'extrême; malentendus qui devraient cesser et qu'on devrait faire cesser $!{ }^{8}$

Il faudrait citer ici toute la lettre pastorale que $\mathrm{Mgr}$ de Sherbrooke a publiée dans son diocèse promulguant la Bulle d'érection canonique de l'Université Laval, pour montrer quel attachement l'ancien curé de St-Jean de Québec entretenait pour le Séminaire de Québec: "C'est au Séminaire de Québec que nous devons l'inestimable bienfait de notre éducation. ${ }^{9}$ Puis il continue:

Comme évêque, nous ne pouvons jamais assez remercier et bénir la Providence divine de ce que l'Université Laval prenant droit de cité parmi les Universités les plus célèbres $d u$ monde catholique, trouve une plus grande garantie de succès dans le haut patronage de son Eminence le Cardinal Préfet de la Propagande. ${ }^{10}$

${ }^{4} \mathrm{Mgr}$ Racine à l'abbé Benj. Pâquet, 16 septembre 1876, Un. 110 no AW.

5 Ibid. no $M$.

${ }^{6}$ L'abbé Benj. Pâquet à l'abbé Bolduc, 17 septembre 1876, Un. 115 -

7 Ibid. L'abbé Alexis Pelletier auteur de la Brochure: \&La réforme chrétienne des études classiques 》 (Montréal, 1875), sur le gaumisme. no $\mathrm{T}$.

$8 \mathrm{Mgr}$ A. Racine à l'abbé Benj. Pâquet, 20 septembre 1876, Un. 115 Evêques de Sherbrooke, vol. I, tome I.

10 Ibid. 
Mgr Racine invite ensuite les jeunes gens de son diocèse à ne pas se contenter de l'instruction reçue dans les Séminaires, «mais ambitionnez l'honneur et l'avantage de suivre les cours de l'Université Laval ».11

Des déclarations aussi peu équivoques allaient-elles calmer les inquiétudes des gens de Québec sur l'Évêque de Sherbrooke? Il le semble: "Je viens de recevoir, écrit T.-E. Hamel, la magnifique lettre Pastorale par laquelle V. G. promulgue dans son diocèse la bulle d'érection canonique de l'Université. Nous l'avons tous lue avec une vraie satisfaction et un vrai bonheur. $\gg{ }^{12}$ Dans une note marginale le Recteur ajoute: "Pour ce qui concerne le Séminaire de Québec, veuillez agréer, Mgr, l'assurance de notre très grande reconnaissance pour les témoignages d'affection et d'attendrissement dont V.G. renouvelle publiquement l'expression en termes si affectueux. ${ }^{13}$

Une atmosphère de confiance va maintenant régner entre Sherbrooke et Québec, en dépit de ce petit mouvement d'impatience: «Un mot seulement à la hâte, écrit l'abbé Benjamin Pâquet à l'abbé Bolduc. Mgr Racine est arrivé à Rome ce matin et il m'a pris toute ma journée. J'ai dû aller deux fois à la Station pour son bagage, et parcourir la ville pour lui trouver un logement. ${ }^{14}$ Mais les inquiétudes allaient reprendre pour de bon quand $\mathrm{Mgr} \mathrm{A}$. Racine entreprit un deuxième voyage à Rome en compagnie de gens moins estimés des autorités du Séminaire de Québec: "On annonce d'une manière certaine le voyage à Rome de Mgr Racine. Il doit porter l'adresse au S. Père à l'occasion de sa cinquantième (année) d'épiscopat. ${ }^{15}$ Et voici le point névralgique: «Il sera accompagné de M. Antonin Martel et Laliberté. Il doit demeurer un mois à Rome. Profitez de l'expérience de Mgr Laflèche, et soyez sur vos gardes. Il en reste toujours quelque chose. Si le règlement du Conseil pouvait être

\footnotetext{
11 Ibid.

12 T.-E. Hamel à Mgr A. Racine, 25 octobre 1876, Un. 115 - no BU. 13 Ibid. no CL.

${ }^{14}$ L'abbé Benj. Pâquet à l'abbé Bolduc, 5 novembre 1876, Un. 117 15 T.-E. Hamel à Benj. Pâquet, 26 janvier 1877. Un. 117 - no A.
} 
parti de Rome quand Mgr Racine y arrivera. » ${ }^{16}$ Les compagnons de Mgr Racine ne sont pas les amis de Laval: «Mgr Racine n'est pas venu ici avant son départ. Tenez-vous à distance de lui, il est si rusé ! et ses compagnons vont l'encourager. ${ }^{17}$ Ses compagnons, qui sont-ils? "Soyez certain que vous n'aurez pas un ami dans cette troupe, pas même M. Laliberté qui a fait la bêtise de signer l'adresse du Cercle Catholique au S. Père. » 18 Écrivant à Mgr Taschereau, son Archevêque, l'abbé Pâquet, en séjour à Rome pour défendre les intérêts de l'Université Laval, disait: «Je sais que M. Laliberté approuve complètement la manière de voir de Mgr Racine et des autres suffragants sur des questions où il sait que V. Grandeur est attaquée. » ${ }^{19}$ En plus si l'on considère la liste des pèlerins qui accompagnent l'Évêque de Sherbrooke à Rome, on est frappé du nombre imposant venant de la région de Montréal. Sur les trente-quatre pèlerins, on peut en compter au moins douze de Montréal ou de la région, et seulement cinq de Québec, en incluant ce bon $M$. Laliberté. ${ }^{20}$ On comprend l'inquiétude des autorités de Québec, à une époque où les Cours de Rome regorgeaient d'accusations contre l'Université Laval.21

Ce voyage de 1877 à Rome effectué par Mgr de Sherbrooke, était-il un voyage d'affaires ? Mgr Racine y avait-il prémédité une attaque contre son Alma Mater ? Ses amis de Québec l'ont bien pensé. Mgr Benj. Pâquet ${ }^{22}$ toujours à Rome, écrivait au Recteur de l'Université Laval: «Je suis convaincu que Mgr Racine nous fera du mal à Rome; je ferai mon possible pour parer les coups. Les siens seront d'autant plus terribles qu'il est regardé à Rome comme un grand ami de l'Université. » ${ }^{23}$ L'Archevêque Taschereau se serait-il donc laissé tromper quand il reçut amica-

16 Ibid.

17 L'abbé Bolduc à l'abbé Benj. Pâquet, 13 avril 1877, Un. 117 - no CD. 18 Ibid. no CK.

${ }^{19}$ L'abbé Benj. Pâquet à Mgr Taschereau, 1 juillet 1877, Un. 118 -

$20 \mathrm{Mgr}$ A. Racine, Cahier de notes de voyages, A.A.S.

21 R. Rumilly, Mgr Laflèche et son temps, (Ed. Zodiaque, Montréal, 1838) passim.

${ }^{22}$ Monsieur Benjamin Pâquet a été, dans l'intervalle, nommé Camérier Secret de Sa Sainteté, Un. 117 - no AA2.

23 Mgr B. Pâquet à T.-E. Hamel, 18 février 1877, Un. 117 - no AA2. 
lement les conseils de l'Évêque de Sherbrooke, comme l'affirmait quelques semaines auparavant M. Bolduc à son ami de Rome: "Mgr l'Archevêque semble un peu plus courageux et je pense que la dernière visite de Mgr Racine lui a fait du bien et l'a affermi dans ses résolutions. Pour peu que ce Seigneur se dévoue à la bonne cause quand il sera à Rome, il fera un grand bien et rendra les autres suffragants impuissants à nuire à l'Archevêque et à mettre le trouble dans le pays. ${ }^{24}$ Mais on sait que Mgr Racine n'était pas allé à Québec immédiatement avant son départ, ${ }^{25} \mathrm{ce}$ qui souleva quelques soupçons. Il y avait plus: Mgr Racine devait présenter au Pape une adresse que M. Laliberté «a fait la bêtise de signer », et qui contenait soi-disant des accusations contre l'Archevêque de Québec: «Tâchez, écrivait l'abbé Bolduc, de voir ce document; il est dans un rouleau ouvert dans une des caisses que j'ai expédiées au R.P. Brichet. C'est un chef-d'œuvre du genre. ${ }^{26}$ Que contenait cette adresse ? Par qui était-elle rédigée ? Les documents que nous avons sous les yeux ne nous en disent rien, sauf que la dite adresse a comme par hasard disparu des malles de Mgr Racine juste au moment où il allait en avoir besoin.

Voici les détails de cet incident banal qui tourne à l'anecdote, mais qui montre l'atmosphère de suspicion dans laquelle on vivait. L'abbé Bolduc écrit de Québec à Mgr B. Pâquet, qu'il a déposé, dans les caisses adressées au R.P. Brichet, l'adresse du Cercle Catholique et il lui conseille de «voir ce document... un chef-d'œuvre du genre ${ }^{2 \tau}$ Ce document et le contenu des caisses devaient être remis à Mgr Racine qui allait se charger de présenter le tout au S. Père.

Or l'adresse avait disparu. Aurait-elle été volée, ou écartée à la douane italienne? Ou bien M. l'abbé Bolduc aurait-il omis

24 L'abbé Bolduc à Mgr Pâquet, 16 février 1877, Un. 117 - no AA.

25 L'abbé Bolduc à Mgr Pâquet, 13 avril 1877, Un. 117 - no CD. Le groupe devait partir de Montréal en train, pour aller prendre le bateau le 12 avril 1877 à New-York. D'après le Cahier de notes de voyages de Mgr Racine. A.A.S.

26 Ibid. Le Père Brichet était supérieur du Séminaire français à Rome, où logeait Mgr Benj. Pâquet, Un. 118 - no CF.

27 L'abbé Bolduc à Mgr Pâquet, 13 avril 1877, Un. 117 - no CD. 
de l'inclure dans les bagages ? M. Laliberté a été vexé et est venu à deux reprises demander au Père Brichet de lui remettre son adresse pensant qu'il la cachait. « Naturellement le P. Brichet a été un peu chagriné de voir qu'il était sous l'impression qu'elle se trouvait entre ses mains. ${ }^{28}$ Mgr Pâquet comme le P. Brichet se défendent bien d'avoir soustrait ce document: «Je vous dirai que je n'ai jamais eu d'autre idée à ce propos qu'elle aurait pu être perdue ou soustraite dans les bureaux de la douane où on a coutume d'ouvrir les caisses afin de vérifier les envois. » ${ }^{29}$ Colère de M. Laliberté !

Quoiqu'il en soit de cette adresse, il semble bien que Mgr Racine n'en ait pas considéré la perte comme sérieuse; ni dans sa correspondance, ni dans son journal de voyage, où il raconte tout en détail, il n'est fait mention de l'adresse du Cercle Catholique au S. Père.

Quels furent les résultats de ce voyage de Mgr de Sherbrooke à Rome? On ne le saura qu'à la révélation des documents renfermés dans les archives du Vatican. Ses amis de Québec, qui ne manquaient pas de flair, croient, pour leur part, que le voyage n'eut pas grand résultat contre l'Université Laval ou l'Archevêque: «D'après ce que je puis voir, Mgr Racine n'a rien fait à Rome. On lui a répondu invariablement que tout était entre les mains du Délégué. » ${ }^{30}$ Pourtant son passage à Rome n'aura pas été sans conséquences. Il aurait agité la question de donner aux Évêques suffragants un droit de surveillance dans la nomination des Professeurs et du Recteur de l'Université. ${ }^{31}$ Puis Mgr Pâquet ajoute dans ce rapport à l'Archevêque :

Mgr Racine est peut-être plus modéré que les Evêques de Rimouski et des Trois-Rivières, mais comme le disait le chanoine Lamarche au Cardinal Angelis, c'est pour ne pas nuire à son frère qu'il désire être élu pour Evêque de Chicoutimi. J'ai déjà eu occasion de dire à V.G. qu'Elle devait se défier de Mgr Racine ; aujourd'hui plus que jamais, j'ai ces raisons de faire la même recommandation. ${ }^{31}$

28 Mgr Benj. Pâquet à l'abbé Bolduc, 30 juin 1877, Un. 118 - no CD.

29 L'abbé Bolduc à Mgr Pâquet, 15 juin 1877 , Un. 118 - no $\mathrm{BO}$.

30 J.-B.-S. Bolduc à Mgr B. Pâquet, 28 juillet 1877 , Un. 119 - no U.

31 Mgr B. Pâquet à Mgr Taschereau, 3 juin 1877, Un. 118 - no AR. 
A la fin de ce chapitre, peut-on clairement affirmer que Mgr Antoine Racine soit devenu un adversaire de l'Université Laval ? Nous ne le croyons pas. En une époque de globalisme politique et doctrinaire, nous croyons même que c'est faire preuve d'un sain jugement d'observer une certaine disponibilité.

Mgr Racine, tout en restant profondément attaché à son Alma Mater, ${ }^{32}$ n'était pas sans se rendre compte que son diocèse s'articulait sur Montréal et qu'il en dépendrait un jour uniquement, tant du point de vue religieux que du point de vue universitaire.

\section{Chapitre II}

\section{MGR RACINE ET LES ACTIVITES POLITIQUES \\ DES PROFESSEURS DE L'UNIVERSITE}

L'Evêque élu est consulté - Différend entre le Séminaire de Québec et l'Assemblée des Evêques - La position de Mgr A. Racine après deux ans d'épiscopat.

Avant même que Mgr Racine fût sacré évêque, un problème s'était posé à l'Université Laval, qui allait déterminer l'intervention des Évêques de la Province Ecclésiastique de Québec: le problème des professeurs de l'Université qui, en marge de leur enseignement, se mêlaient activement de politique, en vue soit d'améliorer leur salaire d'alors, soit de travailler à conquérir les postes honorifiques et lucratifs de députés ou de juges. ${ }^{33}$ Le 17 octobre 1874 - soit la veille du sacre à Québec de Mgr Antoine Racine ${ }^{34}$ - Mgr E.-A. Taschereau, Archevêque de Québec, transmettait au Supérieur du Séminaire québécois trois résolutions adoptées par les Évêques de la Province dans leur réunion du 3 octobre de la même année:

32 « J'ai revu Québec et mes amis; je crois qu'il n'y a qu'un Québec dans le monde, car maintenant je sais ce qu'il en coûte pour se séparer et s'éloigner pour toujours. 》 Mgr Racine à Mgr Pâquet, 1 décembre 1874, Un. 110 - no CV.

33 Mémoire du Séminaire de Québec à Mgr Taschereau, janvier 1876, Un. 112 - no AB.

34 P.-J.-A. Lefebvre, Monseigneur Antoine Racine, p. 9. 
1) Qu'il serait désirable que les professeurs des Universités Catholiques ne se mêlent pas activement de politique.

2) Que les Evêques de cette Province regrettent beaucoup que, dans certaines luttes politiques, leur nom ou celui de quelque institution catholique ait été invoqué sans autorisation pour appuyer certaines personnes ou certaines opinions.

3) Que chaque Evêque soit autorisé à communiquer ces résolutions à qui il jugera à propos..$^{35}$

Or d'après une déclaration écrite de $\mathrm{Mgr}$ Laflèche, au sujet d'un conversation qu'il aurait eue avec l'Évêque de Sherbrooke le 13 juin 1876, Mgr A. Racine aurait été l'un des auteurs de cette demande. Ce qui nous fait croire, qu'avant même d'être sacré évêque, il assistait aux réunions, comme Evêque élu. Voici la déclaration de Mgr Laflèche qui rapporte les paroles de Mgr A. Racine:

Je déclare qu'en Octobre 1874 deux Prêtres du Séminaire de Québec et Professeurs à l'Université, m'ont prié d'intervenir dans l'Assemblée des Evêques à Québec, m'assurant que si les Evêques exprimaient le simple désir que les Professeurs ne se mêlassent pas de politique, le Séminaire accéderait de suite à ce désir. ${ }^{36}$

De fait, on avait consulté Mgr Racine sur ce problème dès le début d'octobre. ${ }^{37}$

L'Évêque élu était d'avis que l'Archevêque pressait trop les termes des résolutions et prétendait «qu'il aurait mieux valu traîner ${ }^{38}$ C'était là, à son avis, un problème que seul le temps pouvait résoudre. Retirer brusquement les Professeurs de la politique, c'était comme ruiner le personnel de l'Université; ainsi le pensait Mgr Racine, ainsi le pensaient les autorités du Séminaire: "Car, il est bien certain que nous n'aurons pour professeurs que des gens sans avenir; ou bien ils nous quitteront aussitôt

\footnotetext{
35 Ibid.

36 Mgr Laflèche à ? 13 juin 1876, Un. 113 - no CL.

37 T.-E. Hamel à Benj. Pâquet, 9 octobre 1874 , Un. 110 - no BL. 38 Ibid.
} 
que la politique leur sourira. ${ }^{39}$ La difficulté a surgi entre l'Université et l'Assemblée des Évêques de ce fait surtout qu'on a pris le désir des Évêques pour un ordre, et qu'on a refusé d'agir par crainte d'intrusion. L'abbé J.-B.-Z. Bolduc optait plutôt pour la temporisation: "C'est un désir, qu'ont exprimé les Évêques dans leur dernière assemblée. » ${ }^{40} \mathrm{Mgr}$ Racine s'en tenait au même propos le 11 janvier 1876, en écrivant au Recteur de l'Université Laval: "Je remarque que vous interprétez ces paroles "Nous recommandons unanimement ${ }^{41}$ comme un ordre donné par les Évêques; mais ces mots veulent dire aussi 'Nous conseillons, nous exhortons'; et c'est le sens qu'il faut leur donner, dans mon humble opinion $\gg^{42}$

L'opinion de l'abbé Bolduc et de Mgr Racine correspondaitelle à la réalité? Nous ne le croyons pas. En septembre 1875, voyant que leur désir n'avait pas été réalisé, l'Assemblée des Évêques transmettait au Supérieur du Séminaire et Recteur de l'Université Laval, par la plume de son jeune secrétaire Mgr Racine, les résolutions suivantes:

Les Evêques de la Province regrettent profondément, dans l'intérêt même de l'Université Laval, que le désir exprimé par eux dans la réunion du 3 octobre dernier n'ait eu aucun résultat pratique, et qu'il ait été complètement déçu dans les dernières élections. En conséquence, ils croient de leur devoir de recommander unanimement au Conseil Universitaire, etc. ${ }^{43}$

Comme quoi nous ne pouvons pas être d'accord avec Mgr Racine sur le sens du mot « recommander ».

Les Évêques de la Province ont réagi là-dessus, d'une toute autre manière que l'Évêque de Sherbrooke, ancien de Laval: «Ils (les Évêques) ne peuvent s'empêcher d'y voir de la part du Séminaire de Québec, le désir clairement exprimé que l'Épiscopat de la Province ne s'immisce aucunement dans ce qui regarde

39 Ibid.; Mémoire du Séminaire de Québec à Mgr Taschereau, Un. 112 - no AB.

40 L'abbé Bolduc à l'abbé Benj. Pâquet, 20 nov. 1874, Un. 110 - no C.

41 Mémoire du Sém. de Québec, Un. 112 - no AB.

$42 \mathrm{Mgr}$ A. Racine à T.-E. Hamel, 11 janvier 1876 , Un. 112 - no AF.

43 Mémoire du Sém. de Québec, Un. 112 - no AB. 
l'Université Laval ».44 Pour eux, cette attitude du Séminaire de Québec ferait savoir aux Évêques que leur institution est sous la régie diocésaine et "ne relevant que de son Ordinaire »." Évidemment Mgr Racine n'est pas ici tout à fait en accord avec ses confrères dans l'Épiscopat. Mais il y viendra, sans négliger "d'être très favorable à l'Université Laval ».46 Il fera adopter la politique de soumettre les observations de l'Assemblée à l'Archevêque en personne, plutôt que directement aux autorités de l'Université: "Si nous croyons utile de faire des observations ou des recommandations, nous les ferons à Mgr l'Archevêque, le Visiteur, et je crois que ce sera mieux pour les uns et pour les autres. ${ }^{47}$

Si les Évêques s'intéressent aussi activement à la politique des Professeurs de l'Université, c'est qu'il y avait là le fameux problème du libéralisme.

L'étude du libéralisme dans la Province de Québec déborde les cadres du présent travail. Qu'il nous soit seulement permis de signaler au passage certains incidents auxquels Mgr Racine a été mêlé. On l'a accusé de se mêler de cette question des Professeurs de l'Université uniquement par intérêt politique, par antipathie contre l'Archevêque reconnu pour un libéral politique, ${ }^{48}$ et par haine du Séminaire de Québec. ${ }^{49}$

Mgr Racine protesta:

L'Evêque de Sherbrooke a fait son cours d'étude au Séminaire de Québec et il est attaché de cœur à cette Institution qui a rendu de si grands services à son pays; son témoignage ne saurait être suspect. Mais il est de son devoir de déclarer à S. E. que par suite des écarts de quelques-uns des professeurs qui se mêlent trop activement de politique, et de l'appui donné par ces Professeurs à ceux qui se sont toujours distingués pour leur hostilité à notre foi et à

${ }^{44} \mathrm{Mgr}$ A. Racine à T.-E. Hamel, 17 janvier 1876, Un. 112 - no AK. 45 Ibid.

$46 \mathrm{Mgr}$ A. Racine à T.-E. Hamel, 25 février 1876, Un. 112 - no BX.

$47 \mathrm{Mgr}$ A. Racine à l'abbé Benj. Pâquet, 7 mars 1876, Un. 112 - no BX.

48 R. Rumilly, Mgr Laflèche et son temps, passim. no AM.

49 M. J.-B.-Z. Bolduc à l'abbé Benj. Pâquet, 21 janvier 1876, Un. 112 
nos Institutions, L'université perd la confiance d'un grand nombre de pères de famille qui éloignent leurs enfants de cette Institution. ${ }^{50}$

On le voit, Mgr Racine, dans ce problème de l'activité politique des Professeurs de l'Université, n'a pas voulu prendre une position nette et catégorique. Il s'en sentait empêché, sans doute, par son attachement profond à l'Université Laval et au Séminaire de Québec ${ }^{\tilde{}} 1$ et par une connaissance plus avertie des réalités concrètes du milieu universitaire; d'autre part il tenait à rester solidaire de ses confrères dans l'Épiscopat. ${ }^{52}$

\section{Chapitre III}

\section{MGR A. RACINE S'OPPOSE À LA FONDATION D'UNE UNIVERSITÉ À MONTRÉAL}

Mgr Racine pense comme l'Archevêque Taschereau - Une deuxième université, un malheur pour Laval — Les Jésuites de Montréal - Mgr A. Racine écrit à Rome - La réponse de Rome - Une succursale de Laval à Montréal.

A Québec, on ne mit pas de temps à inviter le nouvel Évêque de Sherbrooke, à se joindre à la cause de l'Université Laval. On y tenait d'autant plus que le nouveau diocèse allait - par la force des choses - s'articuler sur Montréal. On presse donc Mgr A. Racine d'intervenir auprès du Cardinal Préfet de la Propagande. Il s'agit de convaincre le Préfet d'empêcher la fondation à Montréal d'une deuxième université catholique de la Province ! ${ }^{53}$ Mgr Racine répond à cette invitation et soutient la même thèse que l'Archevêque de Québec. ${ }^{54}$ Dans une lettre de Mgr de Sherbrooke à son ami, l'abbé Benjamin Pâquet, on peut voir nettement sa pensée sur le projet d'une université à Montréal,

50 Mgr Racine à l'abbé Benj. Pâquet, 13 juin 1876, Un. 113 - no CL.

$51 \mathrm{Mgr}$ A. Racine à l'abbé B. Pâquet, 1 janvier 1876 , Un. 112 - no Z; $i d$. à $i d ., 13$ juin 1876 , Un. 113 - no CL.

52 Mgr A. Racine à l'abbé B. Pâquet, 11 janvier 1876 , Un. 112 - no AF ; $i d$. à $i d ., 1$ janvier 1876 , Un. 112 - no Z. no CA.

$53 \mathrm{Mgr}$ A. Racine à (l'abbé Bolduc), 11 novembre 1874, Un. 110 no

54 L'abbé Bolduc à l'abbé B. Pâquet, 20 novembre 1874, Un. 110 - 
projet dont Mgr Ignace Bourget s'est fait le patron: «J'espère que Rome y pensera sérieusement avant de donner une Université à Montréal, et de donner suite au projet dont la conséquence certaine sera la fermeture des portes de l'Université Laval. » ${ }^{55}$

Les opposants à l'université montréalaise se retranchent derrière un argument impressionnant: un certain niveau des études à maintenir. Une seconde Université, croient-ils sincèrement, priverait Laval de ses sujets et de ses finances, entraînant, par ricochet, d'autres et plus graves conséquences. ${ }^{56}$ Devant cette plausible catastrophe, rien de plus naturel que Mgr Racine se réjouisse quand on lui assure que Montréal ne réussira pas à obtenir de Rome la permission demandée: "J'espère qu'il en sera ainsi pour le bien de notre pays. » ${ }^{57}$ Pour la même raison, Mgr l'Évêque de Sherbrooke pourra déclarer qu'il est « au service du Séminaire de Québec et disposé à faire tout ce qui sera en mon pouvoir, auprès de la Cour de Rome, lorsque les MM. du Séminaire trouveront qu'il est à propos de parler et d'agir. $»^{58}$

Mgr Bourget n'était pas seul à travailler en faveur de l'Université de Montréal; les Jésuites y mettaient aussi la main: «Ce que tu me dis des démarches assidues des Jésuites de Rome me fait peine et me fait craindre un peu. Cependant je garde spem contra spem; il me semble que le S.P. ne se décidera jamais à une mesure qui détruira Laval. » ${ }^{59}$ Cette conviction de l'impuissance du Canada français à soutenir deux Universités était fortement ancrée chez les Québecois de l'époque: «Je considère comme un malheur, écrit l'ancien curé de St-Jean de Québec, et comme un grand malheur, l'établissement d'une deuxième Université, à Montréal, parce que c'est ruiner Laval et l'empêcher inutilement de rendre à la Religion et à la Patrie les services no

55 Mgr A. Racine à l'abbé Benj. Pâquet, 1 décembre 1874, Un. 110 -

56 Ibid.

57 Ibid.

$58 \mathrm{Mgr}$ A. Racine à T.-E. Hamel, 20 décembre 1874, Un. $75-$ no 26.

$59 \mathrm{Mgr}$ A. Racine à l'abbé Benj. Pâquet, 21 janvier 1875 , Un. III no $X$; Les Jésuites du Collège Sainte-Marie et l'Université de Montréal, Archives de l'Archidiocèse de Sherbrooke, Section XIII casier B-1 chemise A-2. Nous citerons désormais: AAS, XIII, B-1, A-2. 
qu'il ne cesse de lui rendre depuis sa fondation. ${ }^{60}$ Et dans la même pièce, le même homme affirme qu'une deuxième Université ne peut amener que l'abaissement du niveau des études, et l'encombrement dans les professions libérales. De là l'impatience à recevoir de Rome la réponse 《favorable», c'est-à-dire défavorable aux partisans d'une Université à Montréal. ${ }^{61}$ La réponse finit par sortir du lent engrenage des Congrégations romaines. L'abbé Benjamin Pâquet, en date du 13 février 1876, avertit Mgr Racine que la décision de Rome sur la question universitaire est donnée: «Le jugement est rendu et cette fois c'est pour toujours, il est définitif. $\gg 62$ En voici les points principaux tels qu'énumérés dans la lettre de l'abbé Pâquet:

$1^{\circ}$ La demande de Monseigneur de Montréal est refusée;

$2^{\circ}$ La Succursale est accordée, elle devra être organisée sur les bases proposées par Laval telles qu'énumérées dans la lettre de Mgr Racine au Cardinal Franchi. Montréal en fera tous les frais;

$3^{\circ}$ Il y aura un Conseil Supérieur des Évêques de la Province pour surveiller la foi et les mœurs, tant dans l'Université que dans la Succursale;

$4^{\circ}$ Exhortations à NN. SS. les Évêques d'affilier leurs Collèges à l'Université et d'y diriger leurs jeunes gens. ${ }^{63}$

Puis, avant de parler d'autre chose, l'abbé Pâquet ajoute ce renseignement que le jeune Évêque de Sherbrooke a dû priser: «Je dois vous dire, Monseigneur, que trois ou quatre Cardinaux m'ont parlé d'une manière très élogieuse de votre lettre. ${ }^{64}$

Quelle fut la réaction de Mgr Racine devant la décision de Rome ? Il fallait s'y attendre ! « Je me réjouis de ce jugement parce qu'il assure l'avenir de Laval et qu'il met fin à des contestations plus que désagréables. ${ }^{65}$ Et l'argument du début revient:

$60 \mathrm{Mgr}$ Racine à l'abbé Benj. Pâquet, ibid.

$61 \mathrm{Mgr}$ A. Racine à l'abbé Benj. Pâquet, 27 mars 1875, Un. III no AU; $i d$. à $i d ., 8$ juillet 1875 , Un. III - no CD. no $\mathrm{BN}$.

${ }^{62}$ L'abbé Benj. Pâquet à Mgr A. Racine, 13 février 1876, Un. 112

63 Ibid.

64 Ibid.

$65 \mathrm{Mgr}$ A. Racine à l'abbé Benj. Pâquet, 7 mars 1876 , Un. 112 - no CH. 
« Nous aurons une seule Université Catholique, et le niveau des études, au lieu de diminuer, montera au contraire au grand avantage de la Religion et de la Patrie. Je dis donc de grand cœur: Deo Gratias !»66 Maintenant Mgr I. Bourget ne pourra plus se plaindre, "puisque Rome lui donne l'avantage d'avoir Laval à Montréal même; c'est plus que l'Université qu'il se proposait d'établir. ${ }^{67}$

La Succursale de l'Université Laval à Montréal va-t-elle donner satisfaction aux ambitions des Montréalais ?

Il ne semble pas.

Chaprtre IV

\section{LA SUCCURSALE DE L'UNIVERSITÉ LAVAL À MONTREAL}

L'Ecole Victoria - Rapports avec la Succursale - Le Dr d'Orsonnens - L’illégalité de la Succursale - Mgr A. Racine délégué à Rome pour défendre Laval - Mémoire à Léon XIII - Mgr I. Bourget à Rome - Duel Racine-Bourget La réponse du Saint-Père.

Il existait à Montréal, depuis 1843, une École de Médecine et de Chirurgie. N'ayant pas réussi à s'affilier à l'Université Laval (à cause de l'infériorité des cours donnés à l'École), ${ }^{68}$ celle-ci avait dû, pour décerner ses diplômes, s'affilier à l'Université Victoria, de Cobourg, Ontario. C'est pourquoi on l'appelait l'École Victoria.

Quand Mgr Bourget forma le projet d'installer à Montréal une université catholique indépendante, il fut tacitement convenu que l'École deviendrait la faculté de Médecine de cette Université. ${ }^{69}$ Cependant, sur les entrefaites, l'Université Laval avait obtenu de Rome, par le décret de la Congrégation de la Propagande en date du premier février 1876, et le privilège de demeurer la seule université catholique de la Province de Québec et le droit

66 Ibid.

67 Ibid.

${ }^{68}$ Mémoire présenté par le Séminaire de Québec à NN. SS. les Evêques de la Province de Québec assemblés aux Trois-Rivières, Octobre 1884, AAS. XIII, B-1, A-1.

69 Nous avons ici résumé le chapitre VII de R. Rumilly, op cit. 
d'ouvrir une Succursale de ses cours à Montréal. Après bien des démarches, Mgr Conroy, le Délégué Apostolique, avait obtenu l'affiliation de l'École de Médecine à la Succursale montréalaise de l'Université Laval. ${ }^{70}$ Mais l'accord, péniblement conclu, ne dura guère. Montréal voulait garder toute son autonomie et n'entendait pas céder à l'ingérence de Laval. C'est pourquoi le Dr d'Orsonnens de Montréal décida de rompre avec Laval. Le docteur alléguait l'illégalité de la Succursale; la charte de Laval ne l'autorisait pas explicitement à fonder pareille école hors de Québec et par ailleurs Rome avait déclaré vouloir s'en tenir à la charte. ${ }^{71}$ Le Docteur, dans un mémoire à l'Assemblée des Évêques, réclama de ces derniers une décision. Les Évêques se déclarèrent incompétents. L'École envoya alors son délégué, le Dr d'Orsonnens, à Rome, présenter le mémoire à la Sacrée Congrégation de la Propagande. ${ }^{72}$ Par le même voyage, le Délégué de l'École Victoria se rendit à Londres prendre la consultation d'un avocat, Farrer Herschell, qui conclut à l'illégalité de la Succursale. ${ }^{73}$ L'Université Laval ne se tint pas pour battue. Elle décida d'agir promptement; elle se mit en instance auprès du gouvernement de Québec pour obtenir un rajustement de sa charte aux besoins de l'heure; ${ }^{74}$ en même temps elle décida d'envoyer à Rome deux délégués qui lui obtiendraient gain de cause contre le Dr d'Orsonnens. "Prévoyant que la sanction du Bill ferait enrager les adversaires de l'Université Laval, le Séminaire a député à Rome, Mgr T.-E. Hamel et l'Archevêché (de Québec), Mgr Antoine Racine. » ${ }^{75}$ C'est donc à titre de délégué

70 R. Rumilly, op. cit., p. 135.

71 Ibid., 136, 145. Cette thèse comme nous l'avons signalé plus haut se résumait à ceci: «L'établissement de Laval en dehors de Québec est illégal en droit civil; or la bulle du Saint-Siège relative à la Succursale montréalaise dit qu'elle ne veut en rien déroger à la charte royale; cui in nulla re derogantium volumus; donc le Saint-Siège ne peut sanctionner un établissement contraire à la charte, et cet établissement de Laval à Montréal est aussi nul en droit canonique. $>$

72 Ibid., 136.

73 AAS, XIII, B-1, A-2; Rapport de l'Université Laval pour années 1879-1885, p. 12.

74 R. Rumilly, op. cit., p. 163 et 149, L'abbé P. Méthot à Mgr Racine, 21 juin 1881, AAS, XIII, B-1, A-2.

75 Journal de Mgr Méthot, Manuscrit 16, p. 28; journal du Séminaire, Volume III, p. 182. 
de l'Archevêque de Québec que Mgr Antoine Racine s'embarque pour aller défendre à Rome les intérêts de l'Université Laval. ${ }^{76}$ On fixe le départ au 2 juillet $1881,{ }^{77}$ mais on se voit contraint de le retarder d'une semaine, soit jusqu'au 9 juillet. ${ }^{78}$ L'Évêque de Sherbrooke ne se cache point la difficulté de sa mission; il sait que d'autres voix se feront entendre à côté de la sienne: "On dit que Mgr Bourget et Mgr Laflèche se rendent à Rome soutenir la position prise par leur seigneurie dans la question de l'Université. » ${ }^{79}$ Puis il ajoute dans cette lettre au Grand Vicaire: "Vous avez essuyé un feu de mitrailleuses allemandes de la pire espèce, et ce n'est pas fini ; il faut s'attendre à d'autres combats et à d'autres surprises; «Honni soit qui mal y pense. ${ }^{80}$

Pouvons-nous suivre les faits et gestes des deux délégués? Nous possédons là-dessus deux documents écrits de la main de Mgr Racine; mais le second a véritablement pour auteurs les autorités du Séminaire de Québec. Le premier de ces documents, c'est un itinéraire de voyage du 9 juillet au 18 octobre 1881, où nous voyons que les délégués n'ont pas perdu leur temps. ${ }^{81}$ L'autre pièce est le Mémoire de l'Université Laval présenté à Sa Sainteté le Pape Léon XIII, le 2 août $1881 .^{82}$

Voici, selon le Mémoire de l'Université présenté au Pape, comment on y expose le problème de la légalité de la Succursale. En tête un court historique de l'Université Laval elle-même, fondée à Québec par le Séminaire de Québec, à la demande de tous les Évêques de la Province de Québec, en 1852. Le 8 décembre de la même année, concession d'une charte civile, par la Reine Victoria d'Angleterre. Après un essai de vingt années, érection

76 T.-E. Hamel à Mgr A. Racine, 28 juin 1881, AAS, XIII, B-1, A-2; Mgr A. Racine à T.-E. Hamel, 29 juin 1881, Un. 134 - no BE.

77 L'abbé P. Méthot à Mgr Racine, 28 juin 1881 ; AAS, XIII, B-1, A-2.

78 L'abbé Hamel à Mgr A. Racine, télégramme, 28 juin 1881, Un. 134 - no BE.

79 Mgr A. Racine à l'abbé T.-E. Hamel, 29 juin 1881, Un. 134 - no BE. 80 Ibid.

81 Cet itinéraire est conservé à Un. 134 - no BS. Nous en donnons le texte au long dans Appendice I, à la fin du présent travail; voir aussi AAS, VII, A-1, G-10.

82 Un. 135 - no K. Nous en donnons le texte complet dans Appendice II, à la fin du présent travail. Un. 135 - no S. Voir Appendice III; Mgr A. Racine à Léon XIII, 10 septembre 1881, Un. 135 - no AD. 
canonique de cette Université, désignée sous le nom de Université Laval, par un Décret signé du Pape Pie IX, le premier février 1876. Mgr Racine s'applique ensuite à faire comprendre au Pape que le Séminaire de Québec n'a accepté de faire cette fondation, qui exige des dépenses énormes, qu'à la condition que cette Université reste la seule dans la Province de Québec «tant que le nombre de ceux-ci (les catholiques) ne suffirait pas pour soutenir deux universités ». Or, dix ans à peine après la fondation, en 1862, Mgr Bourget de Montréal sollicite la fondation d'une Université indépendante pour son diocèse. A quatre reprises, la question est réglée à Rome, toujours dans le sens désiré par l'Université Laval. Mais, en 1876, la Propagande émet un décret, approuvé dans toutes ses parties par le Souverain Pontife, qui règle définitivement le problème. Ce décret refuse, pour le moment, à Montréal la permission d'ouvrir une Université indépendante, mais accorde, conseille la fondation à Montréal d'une Succursale de l'Université Laval.

Le décret a été exécuté sous l'autorité du Délégué Apostolique, Mgr Conroy, et avec l'assentiment de tous les Évêques de la Province. La Succursale a fonctionné pendant trois ans, malgré des obstacles de tous genres.

Enfin, les adversaires, à bout de ressources, crurent trouver dans le silence de la Charte Royale (silence relatif au droit de fonder une Succursale en dehors de Québec), un moyen d'attaquer la légalité de la Succursale. Au lieu d'essayer en bons catholiques, de briser ces difficultés s'ils les croyaient réelles, ils les exagérèrent pour tâcher d'annuler par là l'effet du Décret.

Nous avons raconté plus haut les démarches de l'École Victoria en vue de faire accepter à Rome et à Londres la thèse de l'illégalité. Mgr Racine pilotait habilement sa barque; il a démontré l'injustice des adversaires de Laval; il allait, après cela, toucher un point qui ne pourrait laisser indifférents ni le Pape ni les Congrégations. Travailler contre l'Université Laval c'était faire triompher le protestantisme au Canada: «Ils (les adversaires de Laval) prévinrent le Ministre des Colonies à Londres, mettant en avant les intérêts protestants, pour l'engager à ne 
rien accorder à l'Université Laval. » Puis les autorités de l'Université n'ont fait qu'obéir au Décret et par conséquent au Pape, tandis qu'à Montréal on a méprisé le même Décret; on a tâché de le saboter par un procès où l'Université Laval s'est vue accuser d'avoir agi dans l'illégalité.

Bien que l'Université Laval n'ait jamais eu de doute sur la légalité de sa position, cependant pour ôter aux adversaires leurs prétextes d'attaquer, elle demande avec l'appui de tous les Evêques de la Province, une charte additionnelle en Angleterre. Comme le procès dont on avait fait la menace devait porter sur l'interprétation de la Charte même, le Ministre des Colonies ne crut pas devoir conseiller à la Reine d'intervenir et il insinua que cette question était plutôt du ressort des gouvernements provinciaux.

L'Université Laval s'adressa donc à la Législature de la Province de Québec pour obtenir ce qu'elle souhaitait.

Nous trouvons dans l'ouvrage de R. Rumilly, Mgr Laflèche et son temps, la teneur de ce projet de loi présenté à la Chambre le 3 juin 1881 :

1. L'Université Laval est autorisée à multiplier ses chaires d'enseignement dans les arts et autres facultés, dans les limites de la Province de Québec.

2. Le présent acte viendra en force le jour de sa sanction. ${ }^{83}$

Le projet de loi a été voté au comité des bills privés, à une majorité de 5 . C'est-à-dire 9 contre 4 , le 21 juin $1881 .{ }^{84}$

Et nous revenons au plaidoyer de Mgr Racine. L'évêque note le scandale provoqué par les écrits de Mgr Bourget «contre les avis de l'Évêque diocésain (Mgr Fabre) lequel ne faisait que suivre les décrets du St-Siège. » Cette intervention donna, selon le délégué de l'Archevêque de Québec, à une partie du clergé du diocèse de Montréal (antipathique au nouvel Évêque parce que celui-ci a exécuté les ordres du St-Siège, contrairement aux vues de l'ancien Évêque), ${ }^{85}$ l'audace d'agir ouvertement et publiquement contre les directives de son Évêque.

83 R. Rumilly, op. cit., p. 163 et 149.

84 L'abbé P. Méthot à Mgr A. Racine, 21 juin 1881, AAS, XIII, B-1, A-2; T.-E. Hamel à Mgr Racine, 28 juin 1881, AAS, XIII, B-1, A-2.

${ }^{85} \mathrm{La}$ parenthèse est de Mgr Racine. 
Enfin, l'Évêque de Sherbrooke met le Pape au courant de la sanction du projet de loi dont il a été question plus haut, puis il suggère à Sa Sainteté les remèdes qui s'imposent:

$\mathrm{Du}$ reste, toutes les difficultés eussent été levées du coup, sans agitation, si l'Evêque des Trois-Rivières ne se fût pas séparé de ses vénérables collègues.

Bien que Mgr Laflèche soit devenu ainsi la première cause de toute la tempête, il faut bien y joindre, comme cause secondaire, mais très efficace, l'intervention de Mgr Bourget contre les ordres de son successeur qui est aussi son Evêque.

Les conséquences de cette intervention sont que le troupeau fidèle erre à l'aventure, ne sachant où se trouve la vérité. Il demande la paix et la lumière.

On aperçoit ce que Mgr Racine veut obtenir : une solide, énergique et prompte approbation du St-Siège de la conduite de l'Université Laval et une condamnation non équivoque des adversaires, personnifiés surtout par les Évêques de Montréal et des TroisRivières. La mise à pied de Mgr Laflèche (Mgr Bourget avait déjà démissionné motu proprio), Mgr Racine la demandera avec encore plus de netteté dans une lettre à M. T.-E. Hamel: "Ce qui est opportun pour Mgr l'Archevêque, c'est de demander au StSiège qu'il mette un frein sûr et ferme à la fureur mitrée ou qu'il lui ôte sa mître administrative. ${ }^{86}$

Les résultats se feront un peu attendre. Nous l'avons déjà observé: Mgr Bourget n'avait pas laissé les gens de Québec renseigner seuls les Congrégations; il s'était rendu à Rome en personne. ${ }^{87}$ L'avaient accompagné M. Dumesnil, l'abbé Villeneuve, auteur de la «Comédie infernale», et le sénateur F.-X. Trudel. ${ }^{88}$ Le 4 septembre, Mgr de Sherbrooke note dans son itinéraire: «Nous rencontrons dans l'escalier du Vatican Mgr Mazotti, se rendant chez le St-Père; il nous dit qu'il faut entendre Mgr Bourget. Désappointement et mauvaise humeur. ${ }^{89}$

$86 \mathrm{Mgr}$ A. Racine à T.-E. Hamel, 10 novembre 1881, Un. 135 - no BH.

87 Itinéraire de Mgr Racine, 2 et 20 septembre 1881, Un. 134 - no BS; Mgr A. Racine à T.-E. Hamel, 29 juin 1881, Un. 134 - no BE; journal de l'abbé P. Méthot, manuscrit 16, p. 28.

$88 \mathrm{Mgr}$ Racine à Mgr Marquis, 4 septembre 1881, Un. 135 - no Z; Manuscrit 16, p. 28; «L'Ecole a envoyé Mgr Ignace Bourget, l'abbé Amédée Dumesnil et le sénateur F.-X. Trudel. »

89 Itinéraire de Mgr A. Racine, 4 septembre 1881, Un. 134 - no BS. 
Mgr Racine est réellement de mauvaise humeur. Dans une lettre à Mgr Marquis, ce jour-là, il lui déclare: «nos affaires ont avancé, mais qui sait si l'arrivée de ces délégués (de Montréal) ne sera pas cause d'un long retard. Il faut avoir ici une grosse dose de patience. ${ }^{90}$ Un duel s'amorce devant les Congrégations romaines, duel qui sera « la risée des autres peuples. » ${ }^{91}$ Le délégué de l'Archevêque de Québec se hâte d'écrire une première lettre au Cardinal Siméoni; il lui expose les conséquences graves d'un retard en la décision romaine. Il demande en même temps à l'Honorable A. Chapleau alors à Rome, d'écrire dans les mêmes termes. ${ }^{92}$

Le 9 septembre, une deuxième lettre au même Cardinal le presse de donner une réponse avant l'ouverture des cours de l'Université Laval. ${ }^{93}$ La réponse du cardinal n'arrivant pas, dès le lendemain, Mgr Racine écrit une lettre au Saint-Père, lui faisant voir «l'urgence d'une prompte réponse ». ${ }^{94}$ Le 13 septembre, dans une visite au Cardinal Siméoni, Préfet de la Propagande, les deux délégués de Laval apprennent « que des lettres favorables sont mises à la poste pour l'Archevêque sur la question Laval et l'influence indue. » ${ }^{95}$ La même journée, au cours d'une visite au Cardinal Sacconi, les délégués de Laval apprennent la substance de la réponse du St-Père. «Deo gratias ! ${ }^{96}$ Puis le 22 septembre, avant de quitter Rome définitivement, ils sont reçus en audience auprès de Sa Sainteté. ${ }^{97}$ Il ne reste plus qu'à avertir l'Archevêque de Québec de la victoire obtenue; ce qui se fera aussitôt. ${ }^{98}$

Mais quelle fut précisément cette réponse du Saint-Père ? Fut-elle aussi «favorable» que le voulait l'Université ?

90 Mgr Racine à Mgr Marquis, 4 septembre 1881, Un. 135 - no Z.

91 Ibid.

92 Itinéraire de Mgr A. Racine, 6 septembre 1881, Un. 134 - no BS. no $\mathrm{AC}$

$93 \mathrm{Mgr}$ A. Racine au Cardinal Simeoni, 9 septembre 1881 , Un. 135

94 Mgr A. Racine à Léon XIII, 10 septembre 1881, Un. 135 - no AD.

95 Itinéraire de Mgr A. Racine, 13 septembre 1881, Un. 135 - no BS.

96 Ibid.

97 Ibid., 22 septembre.

98 Cablogramme de Mgr A. Racine à Mgr Taschereau, 21 septembre 1881, Archives de l'Archevêché de Québec; note certifiée conforme à l'original par l'abbé J.-M. Beauchemin, archiviste; journal de l'abbé P. Méthot, manuscrit 16, p. 38 . 


\section{Chapitre V}

\section{LA LUTTE CONTINUE}

Mgr Bourget à Rome - La lettre du Saint-Père à l'Archevêque de Québec - Remerciements du Séminaire de Québec à Mgr Antoine Racine - Mgr Laflèche à Rome - Mgr Antoine Racine demande un Délégué Apostolique.

La nouvelle décision du Saint-Siège n'a pas désarmé Mgr I. Bourget et ses compagnons. Le jour même où Mgr Racine télégraphiait à l'Archevêque de Québec que la cause de Laval était gagnée, l'abbé Dumesnil de son côté envoyait également son télégramme à Montréal: la décision n'était pas finale. L'abbé terminait sa dépêche par ces mots: «Confiance. Travaillons ferme. » ${ }^{99}$ Inquiet de cette nouvelle parue dans les journaux de Montréal, le Supérieur du Séminaire de Québec écrit à Mgr T.-E. Hamel pour lui demander ce qu'il faut en penser. Mgr A. Racine et le Grand Vicaire ont quitté la Ville Éternelle depuis au-delà d'une semaine. ${ }^{1}$ Le Cardinal Siméoni lui-même fait la réponse : «L'affirmation Dumesnil est dénuée de fondement. » ${ }^{2}$ Tout ceci se passait avant que la lettre du Saint-Père, datée du 13 septembre 1881, parvint par bateau à l'Archevêque de Québec. Voici la teneur de cette lettre:

Le Saint-Père, ayant mis à l'examen la question soulevée de nouveau au sujet de l'Université Laval et de la succursale établie à Montréal, a ordonné expressément, dans l'audience extraordinaire d'hier, ${ }^{3}$ tenue pour traiter uniquement de cette affaire, de signifier à votre Seigneurie que c'est Sa volonté décidée que l'on doit s'en tenir au décret de cette Sacrée Congrégation émané le premier jour de février 1876, et continuer d'y donner exécution.

Votre Seigneurie reste chargée de communiquer cet ordre du Pape à tous ses suffragants. ${ }^{4}$

99 R. Rumilly, op. cit., p. 159. no BS.

1 Itinéraire de Mgr A. Racine, 22 sæptembre et 3 octobre, Un. $134-$

2 Ibid.; R. Rumilly, op. cit., p. 160.

3 Itinéraire de Mgr A. Racine, 13 septembre 1881: «Visite au Cardinal Siméoni; il nous apprend que des lettres favorables sont mises à la poste pour l'Arch. sur les questions Laval ». Un. 134 - no 135.

$\$$ Cette lettre est citée par R. Rumilly, op. cit., p. 160. 
La réponse était claire: Rome n'acceptait point la thèse du Dr d'Orsonnens sur l'illégalité civile et canonique de la Succursale. Le Pape maintenait le décret de 1876. Du côté de Québec, l'on crut la paix rétablie. On le crut également à Rome, dans le groupe des amis de Laval. Le Père Brịchet, supérieur du Séminaire français, s'était intéressé au problème avec ferveur. Il écrivait à Mgr A. Racine le 22 octobre 1881: «J'ai appris avec plaisir que sa Grâce Monseigneur l'Archevêque avait reçu de la Propagande des lettres très satisfaisantes, qui, pour des hommes non passionnés, suffiront amplement pour trancher toutes les difficultés et établir dans votre excellent pays une paix profonde. ${ }^{5}$ Mgr Fabre pria le clergé de Montréal, par circulaire du 9 octobre, de se soumettre de bon cœur à la décision du SaintSiège. ${ }^{6}$

Mgr Taschereau réprouva catégoriquement la possibilité d'une révolte contre le décret de Rome: "Puisque le Saint-Siège ordonne de s'en tenir aux prescriptions sages et prudentes de nos conciles, ce serait témérité et désobéissance grave que de suivre une autre règle. $~^{\top}$

Québec ne manqua point de remercier ses deux délégués à Rome pour la bonne besogne qu'ils y avaient accomplie: « Je viens aujourd'hui au nom du Séminaire et de l'Université, écrit Mgr P. Méthot à Mgr A. Racine, vous remercier de tous les soins que vous avez pris et toute la peine que vous vous êtes donnée pour défendre la cause de l'Université auprès de la $\mathrm{S}$. Congrégation de la Propagande et du Saint-Père lui-même. ${ }^{8}$ Selon le Recteur de l'Université Laval, Mgr Racine a obtenu un bien grand résultat, «quoique nos adversaires feignent de nourrir encore des espérances. $\gg 9$

Non, l'agitation des «adversaires » n'était pas une feinte. Un incident significatif l'allait démontrer. Pour calmer les esprits et défendre son Université, l'archevêque de Québec décida au ConA-2.

5 Rév. P. Brichet à Mgr A. Racine, 22 octobre 1881, AAS, XIII, B-1,

6 R. Rumilly, op. cit., p. 160.

7 Cette lettre est citée dans R. Rumilly, op. cit., p. 160.

$8 \mathrm{Mgr}$ P. Méthot à Mgr A. Racine, 29 octobre 1881, AAS, XIII, B-1, A-2.

9 Ibid. 
seil des Évêques de faire signer, par tous ses confrères dans l'Épiscopat, un blâme à l'adresse des journalistes qui lançaient des accusations contre Laval et, ainsi, désobéissaient au SaintSiège. Mgr Laflèche de Trois-Rivières signa cette déclaration conjointe, mais «quelques jour après, il écrivit à Mgr Taschereau qu'il regrettait et retirait cette signature, pour diverses raisons qu'il allait exposer au Saint-Siège. ${ }^{10}$

Étonnement et scandale pour les amis de Laval! Mgr Méthot écrit à l'Évêque de Sherbrooke:

Je ne saurais rien vous dire de neuf sur la rétractation et le départ de Mgr Laflèche, sinon qu'ici, c'est un vrai scandale. Un Evêque! Un vieillard! Un homme de sa réputation! Tout le monde s'accorde à appeler cela un véritable acte de folie. Et qui le forçait de signer cette déclaration ? Pourquoi ne pas user devant le Conseil Supérieur du droit d'accuser Laval que la déclaration laisse aux Evêques ? ${ }^{11}$

A l'entendre le Père Brichet aurait réglé la question d'une façon plus radicale. Il écrit à Mgr Racine: «Ah ! Je sais ce que je ferais à la place de S.E. le Cardinal Siméoni pour avoir la paix ! Il suffirait de mettre à pied un certain personnage que rien ne pourra soumettre, et tout serait fini et tous les Canadiens pourraient dire en s'abordant « et facta est tranquillitas magna!... ${ }^{12}$ Mgr Racine enchaîne: «La rétractation de Mgr Laflèche fera connaître au Saint-Siège, et prouve que je n'ai rien exagéré et que j'ai dit la vérité sur le compte de notre frère dans l'Épiscopat. » Et l'évêque de Sherbrooke, rendu à bout par la «fureur mitrée », propose à l'Archevêque de Québec de dresser une liste de signatures en vue d'obtenir la démission de Mgr Laflèche. ${ }^{13}$ Sachant les bonnes... dispositions de l'Évêque de Sherbrooke, Mgr le Grand Vicaire de Québec lui demande d'user de son influence à Rome:

10 R. Rumilly, op. cit., p. 161. A-2.

$11 \mathrm{Mgr}$ P. Méthot à Mgr A. Racine, 29 octobre 1881, AAS, XIII, B-1,

12 Rév. P. Brichet à Mgr Racine, 22 octobre 1881, AAS, XIII, B-1, A-2; cù il est question de Mgr des Trois-Rivières qui, soit-disant, stimule un journal de sa ville à lancer des injures à l'Université Laval.

$13 \mathrm{Mgr}$ A. Racine à T.-E. Hamel, 10 novembre 1881 , Un. $135-$ no $\mathrm{BH}$; cf. Mgr A. Racine à Léon XIII, 10 septembre 1881, Un. 135 - no AD. 
Votre Grandeur qui a déjà écrit deux fois, ${ }^{14}$ daignerait-elle le faire une troisième fois à ce sujet, en insistant pour que le Cardinal Préfet déclare nettement et carrément que, tout en n'intervenant pas dans les questions de procès in se, le Saint-Siège n'en regarde pas moins comme contraires à ses désirs, toute démarche tendant à s'opposer directement ou indirectement à l'exécution de ses décrets et en particulier toute mesure ayant pour but de susciter l'équivalent d'une Université distincte à Montréal.

Enfin, si V. G. écrit au Card. Siméoni, ne pourrait-elle pas insister sur l'impossibilité de gouverner la Province de Québec tant que Mgr Laflèche conservera sa mître administrative ?... Un petit coup d'épaule de votre part qui ressemblerait à un motu proprio arraché par les événements, appuierait beaucoup l'Archevêque. ${ }^{15}$

Mgr Racine a-t-il accédé à la demande de son aîné de Québec? Nous n'en savons rien. Seules les Archives du Vatican nous le révèleront dans quelques années. Ce qui semble certain, c'est que le Saint-Père, Léon XIII, reçut très froidement Mgr Laflèche. ${ }^{16}$ Autre fait certain, c'est que le voyage de Mgr Laflèche n'a pas été sans influencer les Congrégations. Mgr Zitelli écrit à l'Archevêque de Québec: le mémoire que Mgr d'Ottawa et $\mathrm{Mgr}$ Laflèche ont porté à tous les cardinaux de la Sacrée Congrégation, sera discuté dans une assemblée de tous les Évêques de la Province. ${ }^{17}$

Certains Cardinaux ont prévenu Québec de ne pas s'inquiéter de la présence à Rome de Mgr des Trois-Rivières. L'Archevêque de Québec ne laisse pas d'entretenir quelque inquiétude: « Je me demande pourquoi alors veut-on que le fameux mémoire soit discuté avec tous les évêques réunis ? 》Mgr Taschereau y voit même le danger d'une division dans le corps épiscopal: «J'ai écrit au

14 \& J'ai écrit deux lettres au Card. Préfet depuis mon retour à Sherbrooke. 》 Mgr Racine à T.-E. Hamel, 10 novembre 1881, Un. 135 - no BH.

15 T.-E. Hamel à Mgr A. Racine, 15 novembre 1881, AAS, XIII, B-1, A-2. (Les soulignés sont de l'auteur).

16 R. Rumilly, op. cit., p. 168; également un document à AAS, XIII, B-1, A-2, où sont racontées la mercuriale que sert à Mgr Laflèche le Cardinal Mazotti (20 novembre) et son audience avec le Pape le 25 novembre. Ce document n'est ni signé, ni daté autrement que 1882.

17 Mgr Taschereau à Mgr Racine, 17 mars 1882, AAS, XIII, B-1, A-2. 
Cardinal pour lui signaler les inconvénients et les dangers de cette bataille qui n'aboutira à aucun résultat pratique. ${ }^{18}$ Rome toutefois restera sur ses positions. Encore une fois elle règlera "définitivement 》 la question: «Le Cardinal écrit au Séminaire le 14 septembre. Sa lettre y arrive le premier octobre. La question de la Succursale est réglée définitivement par le décret de 1876, confirmé en 1881. Toutes les autres questions doivent être déférées au Conseil Universitaire (de haute surveillance). » Et l'Archevêque de Québec d'ajouter avec un certain triomphe: «Mgr Laflèche doit donc se présenter devant ce Conseil et apporter les preuves de ses affirmations. Nous l'attendons ! ${ }^{19}$

Devant tant de misères et tant de malentendus de part et d'autre, Mgr A. Racine ne voit plus qu'une solution qui puisse amener une paix décisive: obtenir un guide expérimenté et autorisé. Il l'écrit au Grand Vicaire de Québec: le seul remède à tous ces maux, c'est d'obtenir de Rome un Délégué Apostolique qui ferait l'accord entre les Évêques. La cause du mal, ce sont les Évêques insoumis et en particulier Mgr Laflèche. L'Archevêque de Québec, mis au courant de ce désir de Mgr de Sherbrooke, lui répond: "Vous voudriez $1^{\circ}$ un délégué, $2^{\circ}$ un italien, $3^{\circ} \mathrm{Mgr}$ Persico. Je crois que le temps n'est pas encore venu ... Plus tard ce sera peut-être possible. ${ }^{20}$ Pour Mgr Taschereau les esprits sont trop montés. ${ }^{21}$ Or c'est précisément pour calmer les esprits que Mgr Racine veut obtenir un délégué, ainsi que nous l'avons écrit plus haut. Mgr A. Racine ne sera pas seul à faire cette proposition. Le 7 mars 1882, Mgr Benjamin Pâquet lui mande: « Je suis toujours d'avis qu'un délégué apostolique ferait du bien, en donnant aux Évêques l'appui de son autorité, et de son prestige. » ${ }^{2}$ Il apparaît donc que c'est l'Archevêque de Québec qui voit d'un mauvais œil la présence d'une autorité étrangère. $\mathrm{Mgr}$ A. Racine déclare au Grand Vicaire T.-E. Hamel: «Lorsque, à

18 Id. à $i d .1$ avril 1882, AAS, XIII, B-1, A-2.

$19 I d$. à $i d$., 23 octobre 1882 , AAS, XIII, B-1, A-2.

$20 \mathrm{Mgr}$ A. Racine à T.-E. Hamel, 13 février 1882, Un. 136 - no U. A-2.

$21 \mathrm{Mgr}$ Taschereau à Mgr A. Racine, 15 février 1882, AAS, XIII, B-1, A-2.

22 Mgr Benj. Pâquet à Mgr A. Racine, 7 mars 1882, AAS, XIII, B-1, 
Québec, je lui (à l'Archevêque) ai parlé de la nécessité d'un délégué pour faire exécuter les décisions du Pape, il m'a fermé la bouche tout de suite, en me disant: "J'attends quelque chose de Rome; soyez tranquille. ${ }^{23}$ Mgr Racine a voulu insister ; l'Archevêque lui a fait la même réponse: "Soyez tranquille ». L’Évêque de Sherbrooke conclut: «Je suis tranquille, mais de mauvaise humeur. Fasse le Ciel qu'une lettre du St-Père rende inutile la présence d'un délégué. » ${ }^{24}$ Mais qu'attend de Rome l'Archevêque de Québec?

Nous le verrons dans la seconde et dernière partie de ces pages d'histoire.

Germain LaVallée, ptre.

(à suivre)

${ }^{23} \mathrm{Mgr}$ A. Racine à T.-E. Hamel, 28 février 1882, Un. 137 - no BN. 24 Ibid. 